\begin{tabular}{|c|c|c|c|c|c|c|}
\hline$o$. & & & $a$ & $x$ & $\delta$ & \\
\hline 32 & 1872.12 & $8 \mathrm{~h}$ & $47 \mathrm{~m}$ & $42 \mathrm{~s} .75$ & $-1^{0} 59^{\prime}$ & $33^{\prime \prime} 9$ \\
\hline 33 & 1872.12 & 9 & 1 & 5.75 & -356 & 35.6 \\
\hline 34 & 1872.12 & 9 & 42 & 19.77 & +4013 & 44.6 \\
\hline 35 & 872.12 & 9 & 51 & 48 & +4144 & 30.3 \\
\hline 36 & 1872 & 10 & 8 & 21.45 & +4440 & 48.0 \\
\hline & 1872.16 & & & 21.46 & & 47.6 \\
\hline 37 & $187 \%$ & 10 & 10 & 37.17 & +4423 & 52.7 \\
\hline 88 & & 10 & 14 & & +4432 & 48.8 \\
\hline & 18 & & & 15 . & & 49.9 \\
\hline 39 & 9 & 10 & 17 & 12. & +655 & 52.3 \\
\hline 40 & & 10 & 18 & 56 & +87 & 16.2 \\
\hline 41 & & 10 & 19 & 58 & +642 & 6.0 \\
\hline & 6 & & & 58 & & 5.4 \\
\hline 42 & & 10 & 22 & 25 . & +443 & 54.0 \\
\hline 43 & & 10 & 23 & 42. & +622 & 53.4 \\
\hline & & & & 41. & & 55.0 \\
\hline 44 & & 10 & 24 & 42. & +4811 & $43.6:$ \\
\hline 45 & & 10 & 29 & 12.02 & +-1156 & 26.6 \\
\hline & & & & 12. & & 25.7 \\
\hline 46 & 18 & 10 & 33 & 47. & +554 & 31.8 \\
\hline 47 & & 10 & 34 & 8 & +551 & 15.3 \\
\hline 48 & & 10 & 36 & 49 . & -824 & 35.3 \\
\hline & & & & 49 & & 38. \\
\hline 49 & & 11 & 11 & 30 . & +919 & 41.1 \\
\hline 50 & & 11 & 28 & 53. & +1153 & 49.9 \\
\hline 51 & & 11 & 49 & 7.35 & +-114 & $=0.8$ \\
\hline & & & & 7.36 & & 0.4 \\
\hline 52 & 31 & 11 & 59 & 37.37 & +1057 & 11.2 \\
\hline & & & & 37.56 & & 13.0 \\
\hline 53 & 1872.19 & 12 & 21 & 52.06 & -23 & 2.3 \\
\hline & & & & 51.90 & & 3. \\
\hline & & & & 51.91 & & 3.6 \\
\hline 54 & & 12 & 51 & 13.11 & -1339 & 8.3 \\
\hline 55 & & 12 & 56 & 9.91 & -1353 & 50.2 \\
\hline & & & & & & 50.9 \\
\hline 56 & .30 & 13 & 30 & 57.90 & -559 & 55.4 \\
\hline & & & & & & 57.0 \\
\hline 57 & 1872.30 & 14 & 37 & 14.39 & -554 & 28.1 \\
\hline & & & & & & 27.8 \\
\hline 58 & 1872.67 & 20 & 5 & 19.42 & +2131 & 56. \\
\hline
\end{tabular}

\begin{tabular}{|c|c|c|c|c|c|}
\hline \multirow{2}{*}{$\begin{array}{r}\text { No. } \\
59\end{array}$} & \multirow{2}{*}{$\begin{array}{l}\text { Epoche. } \\
1872.65\end{array}$} & \multicolumn{3}{|r|}{$\alpha$} & \multirow{2}{*}{$\begin{array}{c}\delta \\
+21^{0} 29^{\prime} 45^{\prime \prime} .85\end{array}$} \\
\hline & & $20 \mathrm{~h}$ & & $45^{\mathrm{s}} .28$ & \\
\hline & 1872.65 & 20 & 22 & 48.23 & $-17 \quad 18 \quad 57.6$ \\
\hline & 1872.67 & & & 48.15 & 58.7 \\
\hline \multirow[t]{2}{*}{61} & 1872.65 & 20 & 30 & 39.05 & +252624.1 \\
\hline & 1872.67 & & & 39.10 & 23.4 \\
\hline 62 & 1872.67 & 20 & 34 & 54.13 & +2254.5 \\
\hline \multirow[t]{2}{*}{63} & 1872.65 & 21 & 16 & 40.13 & +305448.7 \\
\hline & 1872.67 & & & 40.00 & 48.4 \\
\hline \multirow[t]{2}{*}{64} & 1872.65 & 21 & 27 & 30.34 & $\begin{array}{lll}-4 & 1 & 4.4\end{array}$ \\
\hline & 1872.67 & & & 30.26 & 6.8 \\
\hline \multirow[t]{2}{*}{65} & 1872.65 & 21 & 37 & 24.57 & +325749.5 \\
\hline & 1872.67 & & & 24.37 & 48.3 \\
\hline 66 & 1872.65 & 22 & 6 & 17.75 & $+3522 \quad 1.1$ \\
\hline 67 & 1872.65 & 22 & 17 & 9.85 & $+36 \quad 0 \quad 41.8$ \\
\hline 68 & 1872.67 & 22 & 19 & 57.55 & $+36 \quad 5 \quad 44.3$ \\
\hline 69 & 1872.65 & 22 & 48 & 11.59 & +373144.5 \\
\hline
\end{tabular}

Die vorstehenden Sterne bexiehen sich theils auf hiesige Refractorbeobachtungen, theils sind dieselben auf Wunsch mehrerer auswärtiger Astronomen bestimmt.

Die von No. $40,42,48$ in der vorjährigen Publication gegebenen Bcobachtungen weichen alle stark in demselben Sinne in Declination ab. Damals sind vorher Zonen beobachtet worden und scheint der Nadirpunct sich vom Ende der Zonen bis zum Beginn der Vergleichsternbeobachtungen stark geändert 7.4 haben; nach Ablauf der letzteren ist das Nadir nicht auf's Nene bestimmt worden. Sobald als möglich werden diese Sterne nochmals beobachtet und dann unmittelbar publicirt werden, vorläufig scheint es am gerathensten, jene 3 in voriger Publication gegebenen Beobachtungen gänzlich zu verwerfen. Die Rectascensionen stimmen zwar innerhalb der Beobachtungsfehler, zeigen indessen anch alle $\mathbf{A b -}$ weichungen in demselben Sinn, welche anf cine entsprechende Azimuthänderung deuten.

Vou den Sternen No. 1, 7, 10, 12, 18, 19, 22, 24, $26,28,32,33,34,35,40,42,44,48,62,65,66,67$, 69 sind bereits in den früheren Publicationen Beobachtungen mitgetheilt.

\title{
Beobachtungen des Ganges der mit Barometercompensation versehenen Pendeluhr Knoblich No. 1813.
}

Auf meine Veranlassung brachte Herr Th. Knoblich gegen Ende des Jahres 1871 an einer so eben fertig gewordenen Uhr mit Quecksilberpendel ein Barometerrohr an, dessen innere Weite nach den von Bessel gegebenen Vorschriften zu 0.5 Par. Linie berechnet war. Die Uhr wurde am 29. December in einem Raume der hiesigen 
Sternwarte aufgestellt, und nachdem einige Tage über dem Corrigiren des Ganges vergangen waren, wurden von 1872 Jannar 11 an regelmässige Beobachtungen angestellt. Die Resultate derselben sind folgende:

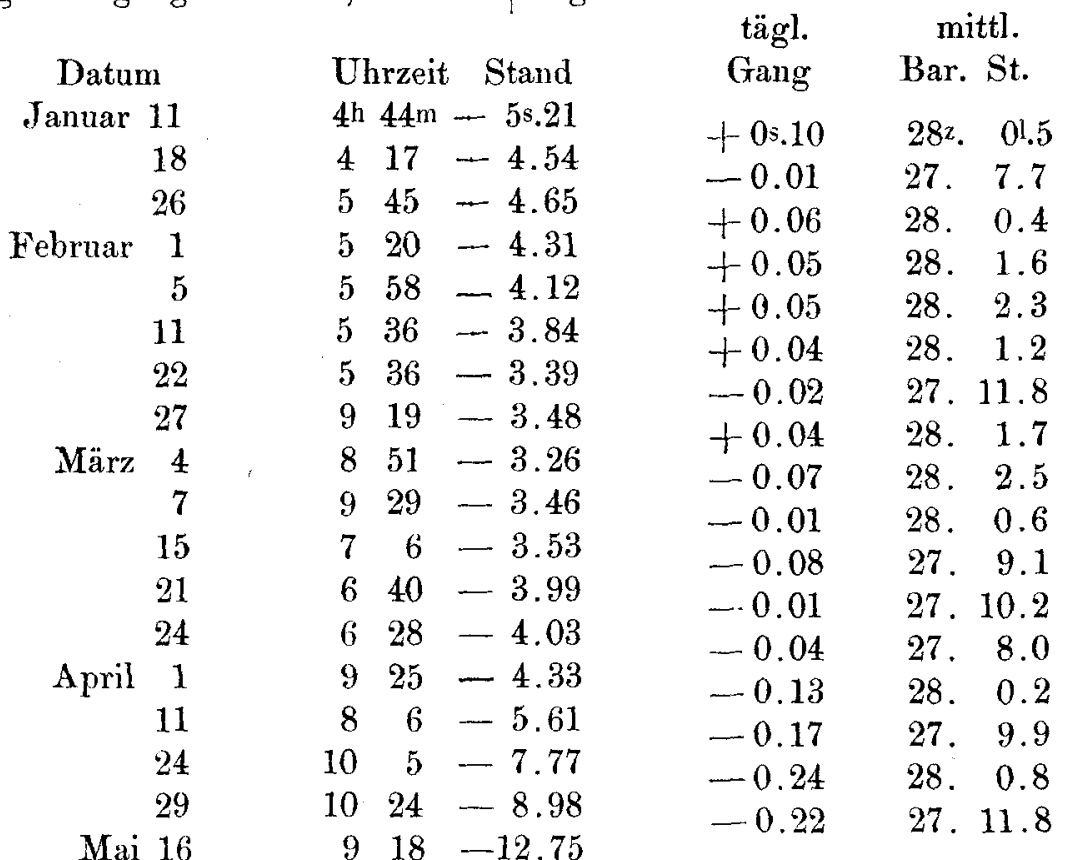

Hiernach fand sich der Uhrgang bei dem niedrig- bringen des Barometers etwas zu stark geworden war, sten Barometerstande von $27 \mathrm{z} 71.7 \mathrm{zu}-0^{\mathrm{s}} .01$, bei dem höchsten Barometerstande von $28 \mathrm{z} 21.5 \mathrm{zu}-0 \mathrm{~s} .07$; es scheint demnach die Compensation gegen Aenderungen des Luftdrucks genau getroffen zu sein. Dagegen zeigt der im April und Mai etwas mehr negativ werdende Uhrgang, dass die Wärmecompensation durch das Anwie auch in der Natur der Sache liegt, - ein Uinstand, welcher durch Herausnehmen einer Kleinigkeit Quecksilber leicht beseitigt werden kann. Ich konnte die Untersuchung der Uhr nicht weiter verfolgen, weil die Ablieferung derselben von ihrem Käufer verlangt wurde. Altona 1373, Febr. 5.
C. F. W. Peters.

\section{Literarische Anzeige.}

Handbuch der Mathematik, Physik, Geodäsie und Astronomie. Von Dr. Rudolph Wolf, Professor in Zürich. Mit zahlreichen in den Text eingedruckten Holzschnitten. 2 Bände. Zürich, Friedrich Schulthess 1872.

Die vier verschiedenen Zweige der Wissenschaft, welche der Verfasser in seinem bereits in $\mathrm{Nr} .1789$ dieser Zeitschrift angekündigten, jetzt vollständig erschienenen Werke behandelt, sind unter einander so innig verbunden, dass Niemand, der sich mit einem von ihnen hauptsächlich beschäftigt, der andern wird entrathen können. Je mehr aber bei den Fortschritten, welche jeder dieser Zweige in Lanfe der Zeiten erlangt, die Kraft für den Einzelnen schwindet, sie sämmtlich mit einiger Vollständigkeit zu umfassen, um so nützlicher werden bei dem häufigen Hinübergreifen der einen in die andere Wissenschaft encyclopädische Werke, in denen das Wesentliche leicht nachgeschlagen werden kann. Mit Recht ist man mehr davon zurückgekommen, derartige Werke über ein gar zu weites Feld auszudehnen, wodurch naturgemäss zı ihrer Vollendung eine grosse Anzahl von Jahren nöthig wird, so dass beim Erscheinen späterer Bände die ersten bereits veraltet sind. Von ungleich grösserer Bedeutung sind die mathematischen, physicalischen u. a. Wörterbücher, welche bei nicht zu grossem Umfange, also leicht in neuen Auflagen herstellbar, das Hauptsäehlichste der in ihnen behandelten Wissenschaft in alphabetischer Folge der einzelnen Artikel darlegen, und je nach ihrer Ausdehnung in grösserer oder geringerer Vollständigkeit ausführen. Diesen jetzt vielfach gebräuchlichen Weg, die einzelnen Artikel in alphabetischer Reihenfolge zu ordnen, hat der Verfasser in seinem Handbuche nicht eingeschlagen, sondern vorgezogen, das Buch in natürlicherer Weise nach den in ihm behandelten Gegenständen in mehrere Abschnitte zu trennen, deren jeder einen 